\title{
Positive solutions and monotone iterative sequences for a fractional differential equation with integral boundary conditions
}

Yongping Sun ${ }^{1 *}$ and Yan Sun ${ }^{2}$

\section{"Correspondence:}

sunyongping@126.com ${ }^{1}$ College of Electron and

Information, Zhejiang University of Media and Communications, Hangzhou, Zhejiang 310018, China Full list of author information is available at the end of the article

\begin{abstract}
Using a monotone iterative method combined with some inequalities associated with the Green's function, we investigate the existence of positive solutions for a fractional differential equation with integral boundary conditions. In addition, two examples are given to illustrate the results.
\end{abstract}

MSC: 34A08; 34B10; 34B15

Keywords: positive solution; fractional differential equations; boundary value problems; integral boundary conditions; monotone iterative method

\section{Introduction}

This paper discusses the existence and iterative method of positive solutions for the following nonlinear fractional differential equations with integral boundary condition:

$$
\left\{\begin{array}{l}
D_{0^{+}}^{\alpha} u(t)+q(t) f(t, u(t))=0, \quad 0<t<1, \\
u^{(j)}(0)=0, \quad 0 \leq j \leq n-2, \quad u(1)=\mu \int_{0}^{1} u(s) d s
\end{array}\right.
$$

where $\alpha \in(n-1, n]$ is a real number and $n \geq 3$ is an integer, $\mu$ is a parameter and $0 \leq \mu<\alpha$, $D_{0^{+}}^{\alpha}$ is the standard Riemann-Liouville fractional derivative of order $\alpha$. A function $u$ is called a positive solution of the problem (1.1) if $u(t)$ satisfies $(1.1)$ and $u(t)>0$ on $(0,1)$.

Fractional differential equations arise in many engineering and scientific disciplines such as the mathematical modeling of systems and processes in the fields of physics, chemistry, aerodynamics, electro-dynamics of a complex medium, polymer rheology, and so on. Recently, the subject of fractional differential equations has gained much more importance and attention. Some excellent work in the study of fractional differential equations can be found in [1-22] and the references cited therein. Integral boundary conditions have various applications in chemical engineering, thermo-elasticity, population dynamics, and so on. Boundary value problems for fractional differential equations with integral boundary conditions are very interesting and largely unknown. Recently, by using GuoKrasnoselskii's fixed point theorem, Cabada and Wang in [5] investigated the existence of positive solutions for the fractional boundary value problem

$$
\left\{\begin{array}{l}
{ }^{C} D_{0^{+}}^{\alpha} u(t)+f(t, u(t))=0, \quad 0<t<1, \\
u(0)=u^{\prime \prime}(0)=0, \quad u(1)=\lambda \int_{0}^{1} u(s) d s,
\end{array}\right.
$$

(02014 Sun and Sun; licensee Springer. This is an Open Access article distributed under the terms of the Creative Commons Attribution License (http://creativecommons.org/licenses/by/2.0), which permits unrestricted use, distribution, and reproduction in any medium, provided the original work is properly cited. 
where $2<\alpha \leq 3,0<\lambda<2,{ }^{C} D_{0^{+}}^{\alpha}$ is the Caputo fractional derivative and $f:[0,1] \times[0, \infty) \rightarrow$ $[0, \infty)$ is a continuous function. Karakostas [10] provided sufficient conditions for the nonexistence of solutions of the boundary-value problems with fractional derivative of order $\alpha \in(2,3)$ in the Caputo sense,

$$
\left\{\begin{array}{l}
{ }^{C} D_{0^{+}}^{\alpha} u(t)+f(t, u(t))=0, \quad 0<t<1, \\
u(0)=u^{\prime}(0)=0, \quad u(1)=\lambda \int_{0}^{1} u(s) d s .
\end{array}\right.
$$

Motivated by the works mentioned above, our purpose in this paper is to show the existence and iteration of positive solutions to the problem (1.1) by using a monotone iterative method. The method used in this paper is different from that used in [20]. We not only obtain the existence of positive solutions, but also give two iterative schemes approximating the solutions, and the iterative scheme starts off with a known simple function or the zero function, which is interesting because it gives a numerical method to compute approximate solutions. The monotone iterative method has been successfully applied to boundary-value problems of integer-order ordinary differential equations (see [23-27] and the references therein). To our knowledge, there is still little utilization of the monotone iterative method to study the existence of positive solutions for nonlinear fractional boundary-value problems. So, it is worth investigating the problem (1.1) by using monotone iterative method.

\section{Preliminaries}

Let us recall some basic definitions on fractional calculus.

Definition 2.1 ([28, 29]) The Riemann-Liouville fractional derivative of order $\alpha>0$ of a continuous function $h:[0, \infty) \rightarrow \mathbb{R}$ is defined to be

$$
D_{0+}^{\alpha} h(t)=\frac{1}{\Gamma(n-\alpha)}\left(\frac{d}{d t}\right)^{n} \int_{0}^{t}(t-s)^{n-\alpha-1} h(s) d s, \quad n=[\alpha]+1,
$$

where $\Gamma$ denotes the Euler gamma function and $[\alpha]$ denotes the integer part of number $\alpha$, provided that the right side is pointwise defined on $(0, \infty)$.

Definition 2.2 ([28, 29]) The Riemann-Liouville fractional integral of order $\alpha$ is defined as

$$
I_{0+}^{\alpha} h(t)=\frac{1}{\Gamma(\alpha)} \int_{0}^{t}(t-s)^{\alpha-1} h(s) d s, \quad t>0, \alpha>0,
$$

provided the integral exists.

In [17], the author obtained the Green's function associated with the problem (1.1). More precisely, the author proved the following lemma.

Lemma 2.1 ([17]) Let $h \in C[0,1]$ be a given function, then the boundary-value problem

$$
\left\{\begin{array}{l}
D_{0^{+}}^{\alpha} u(t)+h(t)=0, \quad 0 \leq t \leq 1, \\
u^{(j)}(0)=0, \quad 0 \leq j \leq n-2, \quad u(1)=\mu \int_{0}^{1} u(s) d s
\end{array}\right.
$$


has a unique solution,

$$
u(t)=\int_{0}^{1} G(t, s) h(s) d s
$$

where

$$
\begin{aligned}
& G(t, s)=H(t, s)+\frac{\mu t^{\alpha-1}}{(\alpha-\mu) \Gamma(\alpha)} s(1-s)^{\alpha-1}, \quad t, s \in[0,1], \\
& H(t, s)=\frac{1}{\Gamma(\alpha)} \begin{cases}t^{\alpha-1}(1-s)^{\alpha-1}-(t-s)^{\alpha-1}, & 0 \leq s \leq t \leq 1, \\
t^{\alpha-1}(1-s)^{\alpha-1}, & 0 \leq t \leq s \leq 1 .\end{cases}
\end{aligned}
$$

Obviously,

$$
G(t, s)=\frac{1}{(\alpha-\mu) \Gamma(\alpha)}\left\{\begin{array}{c}
t^{\alpha-1}(1-s)^{\alpha-1}(\alpha-\mu+\mu s)-(\alpha-\mu)(t-s)^{\alpha-1}, \\
0 \leq s \leq t \leq 1, \\
t^{\alpha-1}(1-s)^{\alpha-1}(\alpha-\mu+\mu s), \\
0 \leq t \leq s \leq 1,
\end{array}\right.
$$

and $G(t, s)$ is continuous on the unit square $[0,1] \times[0,1]$.

Lemma $2.2([16])$ The function $H(t, s)$ defined by (2.2) has the following properties:

$$
t^{\alpha-1}(1-t) \frac{s(1-s)^{\alpha-1}}{\Gamma(\alpha)} \leq H(t, s) \leq \frac{s(1-s)^{\alpha-1}}{\Gamma(\alpha-1)}, \quad t, s \in[0,1] .
$$

Lemma 2.3 The Green's function $G(t, s)$ defined by $(2.1)$ has the following properties:

$$
\begin{aligned}
& 0 \leq G(t, s) \leq \frac{t^{\alpha-1}}{(\alpha-\mu) \Gamma(\alpha)}(1-s)^{\alpha-1}(\alpha-\mu+\mu s), \quad t, s \in[0,1], \\
& p(t) g(s) \leq G(t, s) \leq g(s), \quad t, s \in[0,1],
\end{aligned}
$$

where

$$
\begin{aligned}
& g(s)=\frac{(\alpha-1)(\alpha-\mu)+\mu}{(\alpha-\mu) \Gamma(\alpha)} s(1-s)^{\alpha-1}, \quad s \in[0,1], \\
& p(t)=\frac{\alpha t^{\alpha-1}(1-t)}{(\alpha-1)(\alpha-\mu)+\mu}, \quad t \in[0,1] .
\end{aligned}
$$

Proof It is obvious from (2.3) that the right inequality of (2.5) holds. Relation (2.4) implies that $H(t, s) \geq 0$. Thus by (2.1) we know that the left inequality of $(2.5)$ is correct. Now we show that (2.6) holds. In fact, by (2.1) and (2.4), we have

$$
\begin{aligned}
G(t, s) & =H(t, s)+\frac{\mu t^{\alpha-1}}{(\alpha-\mu) \Gamma(\alpha)} s(1-s)^{\alpha-1} \leq \frac{s(1-s)^{\alpha-1}}{\Gamma(\alpha-1)}+\frac{\mu}{(\alpha-\mu) \Gamma(\alpha)} s(1-s)^{\alpha-1} \\
& =\frac{(\alpha-1)(\alpha-\mu)+\mu}{(\alpha-\mu) \Gamma(\alpha)} s(1-s)^{\alpha-1}=g(s), \quad t, s \in[0,1] .
\end{aligned}
$$


On the other hand, by (2.1) and (2.4), we get

$$
\begin{aligned}
G(t, s) & =H(t, s)+\frac{\mu t^{\alpha-1}}{(\alpha-\mu) \Gamma(\alpha)} s(1-s)^{\alpha-1} \\
& \geq t^{\alpha-1}(1-t) \frac{s(1-s)^{\alpha-1}}{\Gamma(\alpha)}+\frac{\mu t^{\alpha-1}(1-t)}{(\alpha-\mu) \Gamma(\alpha)} s(1-s)^{\alpha-1} \\
& =\frac{s(1-s)^{\alpha-1}}{(\alpha-\mu) \Gamma(\alpha)} \alpha t^{\alpha-1}(1-t)=g(s) p(t), \quad t, s \in[0,1] .
\end{aligned}
$$

Then the proof is completed.

\section{Main results}

Now, we consider the problem (1.1). Obviously, $u$ is a solution of the problem (1.1) if and only if $u$ is a solution of the following nonlinear integral equation:

$$
u(t)=\int_{0}^{1} G(t, s) q(s) f(s, u(s)) d s, \quad t \in[0,1], u \in C[0,1],
$$

where $G(t, s)$ is the Green's function defined by (2.3). For the forthcoming analysis, we need the following assumptions:

(H1) $f:[0,1] \times[0, \infty) \rightarrow[0, \infty)$ is continuous and $f(t, 0) \not \equiv 0$ on $[0,1]$;

(H2) $q:(0,1) \rightarrow[0, \infty)$ is continuous and $0<\int_{0}^{1}(1-s)^{\alpha-1} q(s) d s<\infty$.

The basic space used in this paper is a real Banach space $\mathcal{E}=C[0,1]$ with the norm $\|u\|$, where $\|u\|=\max _{0 \leq t \leq 1}|u(t)|$. Then, define a set $\mathcal{K} \subset \mathcal{E}$ by

$$
\mathcal{K}=\{u \in C[0,1]: u(t) \geq 0, u(t) \geq p(t)\|u\|, t \in[0,1]\} .
$$

It is obvious that $\mathcal{K}$ is a cone. We define the operator $\mathcal{T}: C[0,1] \rightarrow C[0,1]$ by

$$
(\mathcal{T} u)(t)=\int_{0}^{1} G(t, s) q(s) f(s, u(s)) d s, \quad t \in[0,1], u \in C[0,1]
$$

It is clear that the existence of a positive solution for the problem (1.1) is equivalent to the existence of a nontrivial fixed point of $\mathcal{T}$ in $\mathcal{K}$.

Lemma 3.1 $\mathcal{T}$ is a completely continuous operator and $\mathcal{T}(\mathcal{K}) \subseteq \mathcal{K}$.

Proof Applying the Arzela-Ascoli theorem and a standard argument, we can prove that $\mathcal{T}$ is a completely continuous operator. We conclude that $\mathcal{T}(\mathcal{K}) \subseteq \mathcal{K}$. In fact, for any $u \in \mathcal{K}$, it follows from (H1), (H2), and (2.6) that

$$
(\mathcal{T} u)(t)=\int_{0}^{1} G(t, s) q(s) f(s, u(s)) d s \leq \int_{0}^{1} g(s) q(s) f(s, u(s)) d s, \quad t \in[0,1]
$$

which implies that

$$
\|\mathcal{T} u\| \leq \int_{0}^{1} g(s) q(s) f(s, u(s)) d s
$$


On the other hand, by (H1), (H2), and (2.6) we have

$$
(\mathcal{T} u)(t)=\int_{0}^{1} G(t, s) q(s) f(s, u(s)) d s \geq p(t) \int_{0}^{1} g(s) q(s) f(s, u(s)) d s, \quad t \in[0,1],
$$

which together with (3.2) implies

$$
(\mathcal{T} u)(t) \geq p(t)\|\mathcal{T} u\|, \quad t \in[0,1] .
$$

Therefore, $\mathcal{T} u \in \mathcal{K}$. The proof is completed.

For convenience, we denote

$$
\Lambda=\left(\frac{1}{(\alpha-\mu) \Gamma(\alpha)} \int_{0}^{1}(1-s)^{\alpha-1}(\alpha-\mu+\mu s) q(s) d s\right)^{-1} .
$$

By (H2) we know that $\Lambda>0$ is well defined.

Theorem 3.1 Suppose $(\mathrm{H} 1)$ and $(\mathrm{H} 2)$ hold. In addition, we assume that there exists $a>0$, such that

$$
f(t, x) \leq f(t, y) \leq \Lambda a, \quad 0 \leq x \leq y \leq a, t \in[0,1]
$$

where $\Lambda$ is given by (3.3). Then the problem (1.1) has two positive solutions $v^{*}$ and $w^{*}$ satisfying $0<\left\|v^{*}\right\| \leq\left\|w^{*}\right\| \leq a$. In addition, the iterative sequences $v_{k+1}=\mathcal{T} v_{k}, w_{k+1}=\mathcal{T} w_{k}$, $k=0,1,2, \ldots$, converge, in $C$-norm, to positive solutions $v^{*}$ and $w^{*}$, respectively, where $v_{0}(t)=0, w_{0}(t)=a t^{\alpha-1}, t \in[0,1]$. Moreover,

$$
\begin{aligned}
& v_{0}(t) \leq v_{1}(t) \leq \cdots \leq v_{k}(t) \leq \cdots \leq v^{*}(t) \leq w^{*}(t) \leq \cdots \leq w_{k}(t) \leq \cdots \leq w_{1}(t) \leq w_{0}(t), \\
& \quad t \in[0,1] .
\end{aligned}
$$

Remark 3.1 The iterative schemes in Theorem 3.1 start off with the zero function and a known simple function, respectively.

Proof The proof will be given in several steps.

Step 1. Let $\mathcal{K}_{a}=\{u \in \mathcal{K}:\|u\| \leq a\}$, then $\mathcal{T}\left(\mathcal{K}_{a}\right) \subseteq \mathcal{K}_{a}$.

In fact, if $u \in \mathcal{K}_{a}$, then

$$
0 \leq u(s) \leq\|u\| \leq a, \quad s \in[0,1] .
$$

Thus by (2.5) and (3.4), we get

$$
\begin{aligned}
0 & \leq(\mathcal{T} u)(t)=\int_{0}^{1} G(t, s) q(s) f(s, u(s)) d s \\
& \leq \frac{t^{\alpha-1}}{(\alpha-\mu) \Gamma(\alpha)} \int_{0}^{1}(1-s)^{\alpha-1}(\alpha-\mu+\mu s) q(s) f(s, a) d s \\
& \leq \frac{\Lambda a}{(\alpha-\mu) \Gamma(\alpha)} \int_{0}^{1}(1-s)^{\alpha-1}(\alpha-\mu+\mu s) q(s) d s=a, \quad t \in[0,1],
\end{aligned}
$$

which implies that $\|\mathcal{T} u\| \leq a$, thus $\mathcal{T}\left(\mathcal{K}_{a}\right) \subseteq \mathcal{K}_{a}$. 
Step 2. The iterative sequence $\left\{v_{k}\right\}$ is increasing, and there exists $v^{*} \in \mathcal{K}_{a}$ such that $\lim _{k \rightarrow \infty}\left\|v_{k}-v^{*}\right\|=0$, and $v^{*}$ is a positive solution of the problem (1.1).

Obviously, $v_{0} \in \mathcal{K}_{a}$. Since $\mathcal{T}: \mathcal{K}_{a} \rightarrow \mathcal{K}_{a}$, we have $v_{k} \in \mathcal{T}\left(\mathcal{K}_{a}\right) \subseteq \mathcal{K}_{a}, k=1,2, \ldots$. Since $\mathcal{T}$ is completely continuous, we assert that $\left\{v_{k}\right\}_{k=1}^{\infty}$ is a sequentially compact set. Since $v_{1}=\mathcal{T} v_{0}=\mathcal{T} 0 \in \mathcal{K}_{a}$, we have

$$
a \geq v_{1}(t)=\left(\mathcal{T} v_{0}\right)(t)=(\mathcal{T} 0)(t) \geq 0=v_{0}(t), \quad t \in[0,1] .
$$

It follows from (3.4) that $\mathcal{T}$ is nondecreasing, and then

$$
v_{2}(t)=\left(\mathcal{T} v_{1}\right)(t) \geq\left(\mathcal{T} v_{0}\right)(t)=v_{1}(t), \quad t \in[0,1] .
$$

Thus, by the induction, we have

$$
v_{k+1}(t) \geq v_{k}(t), \quad t \in[0,1], k=0,1,2, \ldots
$$

Hence, there exists $v^{*} \in \mathcal{K}_{a}$ such that $\lim _{k \rightarrow \infty}\left\|v_{k}-v^{*}\right\|=0$. By the continuity of $\mathcal{T}$ and equation $v_{k+1}=\mathcal{T} v_{k}$, we get $\mathcal{T} v^{*}=v^{*}$. Moreover, since the zero function is not a solution of the problem (1.1), $\left\|v^{*}\right\|>0$. It follows from the definition of the cone $\mathcal{K}$, that we have $v^{*}(t) \geq p(t)\left\|v^{*}\right\|>0, t \in(0,1)$, i.e. $v^{*}(t)$ is a positive solution of the problem (1.1).

Step 3. The iterative sequence $\left\{w_{k}\right\}$ is decreasing, and there exists $w^{*} \in \mathcal{K}_{a}$ such that $\lim _{k \rightarrow \infty}\left\|w_{k}-w^{*}\right\|=0$, and $w^{*}$ is a positive solution of the problem (1.1).

Obviously, $w_{0} \in \mathcal{K}_{a}$. Since $\mathcal{T}: \mathcal{K}_{a} \rightarrow \mathcal{K}_{a}$, we have $w_{k} \in \mathcal{T}\left(\mathcal{K}_{a}\right) \subseteq \mathcal{K}_{a}, k=1,2, \ldots$. Since $\mathcal{T}$ is completely continuous, we assert that $\left\{w_{k}\right\}_{k=1}^{\infty}$ is a sequentially compact set. Since $w_{1}=\mathcal{T} w_{0} \in \mathcal{K}_{a}$, by (2.5) and (3.4), we have

$$
\begin{aligned}
\left(\mathcal{T} w_{0}\right)(t) & =\int_{0}^{1} G(t, s) q(s) f\left(s, w_{0}(s)\right) d s \\
& \leq \frac{t^{\alpha-1}}{(\alpha-\mu) \Gamma(\alpha)} \int_{0}^{1}(1-s)^{\alpha-1}(\alpha-\mu+\mu s) q(s) f(s, a) d s \\
& \leq \frac{t^{\alpha-1} \Lambda a}{(\alpha-\mu) \Gamma(\alpha)} \int_{0}^{1}(1-s)^{\alpha-1}(\alpha-\mu+\mu s) q(s) d s=a t^{\alpha-1}=w_{0}(t), \quad t \in[0,1] .
\end{aligned}
$$

Thus we obtain

$$
w_{1}(t) \leq w_{0}(t), \quad t \in[0,1]
$$

which together with (3.4) implies that

$$
\begin{aligned}
w_{2}(t) & =\left(\mathcal{T} w_{1}\right)(t)=\int_{0}^{1} G(t, s) q(s) f\left(s, w_{1}(s)\right) d s \\
& \leq \int_{0}^{1} G(t, s) q(s) f\left(s, w_{0}(s)\right) d s=\left(\mathcal{T} w_{0}\right)(t)=w_{1}(t), \quad t \in[0,1] .
\end{aligned}
$$

By the induction, we have

$$
w_{k+1}(t) \leq w_{k}(t), \quad t \in[0,1], k=0,1,2, \ldots
$$


Hence, there exists $w^{*} \in \mathcal{K}_{a}$ such that $\lim _{k \rightarrow \infty}\left\|w_{k}-w^{*}\right\|=0$. Applying the continuity of $\mathcal{T}$ and the definition of $\mathcal{K}$, we can concluded that $w^{*}$ is a positive solution of the problem (1.1).

Step 4. From $v_{0}(t) \leq w_{0}(t), t \in[0,1]$ we get

$$
\begin{aligned}
v_{1}(t) & =\left(\mathcal{T} v_{0}\right)(t)=\int_{0}^{1} G(t, s) q(s) f\left(s, v_{0}(s)\right) d s \\
& \leq \int_{0}^{1} G(t, s) q(s) f\left(s, w_{0}(s)\right) d s=\left(\mathcal{T} w_{0}\right)(t)=w_{1}(t), \quad t \in[0,1]
\end{aligned}
$$

By the induction, we have

$$
v_{k}(t) \leq w_{k}(t), \quad t \in[0,1], k=0,1,2, \ldots
$$

The proof is complete.

Remark 3.2 Certainly, $w^{*}=v^{*}$ may happen and then the problem (1.1) has only one solution in $\mathcal{K}_{a}$.

Corollary 3.1 Suppose that $(\mathrm{H} 1)$ and (H2) hold. Suppose further that $f(t, x)$ is nondecreasing in $x$ for each $t \in[0,1]$ and

$$
\lim _{x \rightarrow+\infty} \max _{0 \leq t \leq 1} \frac{f(t, x)}{x}<\Lambda
$$

Then the problem (1.1) has at least two positive solutions.

\section{Examples}

To illustrate the usefulness of the results, we give two examples.

Example 4.1 Consider the fractional boundary-value problem

$$
\left\{\begin{array}{l}
D_{0^{+}}^{7 / 2} u(t)+3 u^{2}(t)+6 t+1=0, \quad 0<t<1 \\
u(0)=u^{\prime}(0)=u^{\prime \prime}(0)=0, \quad u(1)=\frac{3}{2} \int_{0}^{1} u(s) d s
\end{array}\right.
$$

In this problem,

$$
\alpha=3.5, \quad \mu=1.5, \quad q(t) \equiv 1, \quad f(t, x)=3 x^{2}+6 t+1 .
$$

It is easy to see that (H1) and (H2) hold. If we let $a=2$, by simple computation, we have

$$
\Lambda=\left(\frac{1}{(\alpha-\mu) \Gamma(\alpha)} \int_{0}^{1}(1-s)^{\alpha-1}(\alpha-\mu+\mu s) q(s) d s\right)^{-1}=\frac{45 \sqrt{\pi}}{8}
$$

and

$$
f(t, x) \leq f(t, 2) \leq f(1,2)=19<\frac{45 \sqrt{\pi}}{4}=\Lambda a, \quad(t, x) \in[0,1] \times[0, a]
$$


Then (3.4) is satisfied. Consequently, Theorem 3.1 guarantees that the problem (4.1) has at least two positive solutions $v^{*}$ and $w^{*}$, satisfying $0<\left\|v^{*}\right\| \leq\left\|w^{*}\right\| \leq 2$.

Moreover, the two iterative schemes are

$$
\begin{aligned}
v_{0}(t)=0, \quad t \in[0,1] & \\
v_{k+1}(t)= & \frac{2 t^{5 / 2}}{15 \sqrt{\pi}} \int_{0}^{1}(1-s)^{5 / 2}(4+3 s)\left[3 v_{k}^{2}(s)+6 s+1\right] d s \\
& -\frac{8}{15 \sqrt{\pi}} \int_{0}^{t}(t-s)^{5 / 2}\left[3 v_{k}^{2}(s)+6 s+1\right] d s, \quad t \in[0,1], k=0,1,2, \ldots,
\end{aligned}
$$

and

$$
\begin{aligned}
w_{0}(t)=2 t^{5 / 2}, \quad t \in[0,1] & \\
w_{k+1}(t)= & \frac{2 t^{5 / 2}}{15 \sqrt{\pi}} \int_{0}^{1}(1-s)^{5 / 2}(4+3 s)\left[3 w_{k}^{2}(s)+6 s+1\right] d s \\
& -\frac{8}{15 \sqrt{\pi}} \int_{0}^{t}(t-s)^{5 / 2}\left[3 w_{k}^{2}(s)+6 s+1\right] d s, \quad t \in[0,1], k=0,1,2, \ldots
\end{aligned}
$$

After direct calculations by Matlab 7.5, the second and third terms of the two schemes are as follows:

$$
\begin{aligned}
v_{1}(t)= & \frac{4 t^{5 / 2}}{5 \sqrt{\pi}}\left(\frac{3}{11}-\frac{4}{21} t-\frac{16}{63} t^{2}\right), \quad t \in[0,1] \\
v_{2}(t)= & \frac{4 t^{5 / 2}}{5 \sqrt{\pi}}\left(\frac{3}{11}-\frac{4}{21} t-\frac{16}{63} t^{2}\right)+\frac{64 t^{15 / 2}}{125 \pi^{3 / 2}}\left(\frac{3}{121}-\frac{28}{363} t-\frac{368}{14,553} t^{2}+\frac{27,712}{305,613} t^{3}\right. \\
& \left.-\frac{2,816}{83,349} t^{4}-\frac{1,024}{27,783} t^{5}\right), \quad t \in[0,1]
\end{aligned}
$$

and

$$
\begin{aligned}
w_{1}(t)= & \frac{t^{5 / 2}}{\sqrt{\pi}}\left(\frac{97,252}{415,701}-\frac{16}{105} t-\frac{64}{315} t^{2}-\frac{16,384}{765,765} t^{6}\right), \quad t \in[0,1] \\
w_{2}(t)= & \frac{4 t^{5 / 2}}{5 \sqrt{\pi}}\left(\frac{3}{11}-\frac{4}{21} t-\frac{16}{63} t^{2}\right)+\frac{64 t^{15 / 2}}{135 \pi^{3 / 2}}\left(\frac{591,121,969}{19,200,813,489}-\frac{20,806,384,636}{224,009,490,705} t\right. \\
& -\frac{14,431,376}{509,233,725} t^{2}+\frac{8,246,848}{79,214,135} t^{3}-\frac{2,816}{77,175} t^{4}-\frac{1,024}{25,725} t^{5}-\frac{199,172,096}{35,369,919,585} t^{6} \\
& +\frac{1,832,321,024}{137,549,687,275} t^{7}+\frac{262,144}{187,612,425} t^{8}+-\frac{524,288}{62,537,475} t^{9}+\frac{16,777,216}{65,155,115,025} t^{12} \\
& \left.-\frac{67,108,864}{152,028,601,725} t^{13}\right), \quad t \in[0,1] .
\end{aligned}
$$

Example 4.2 Consider the fractional boundary value

$$
\left\{\begin{array}{l}
D_{0^{+}}^{9 / 2} u(t)+(1-t)^{-3 / 2}\left[e^{u(t)+t+1}+2 u^{2}(t)+4 t+5\right]=0, \quad 0<t<1, \\
u(0)=u^{\prime}(0)=u^{\prime \prime}(0)=u^{\prime \prime \prime}(0)=0, \quad u(1)=2 \int_{0}^{1} u(s) d s .
\end{array}\right.
$$


In this problem,

$$
\alpha=4.5, \quad \mu=2, \quad q(t)=(1-t)^{-3 / 2}, \quad f(t, x)=e^{x+t+1}+2 x^{2}+4 t+5 .
$$

Obviously, $q(t)$ and $f(t, x)$ satisfy the conditions (H1) and (H2). In addition, $f(t, x)$ is increasing in $x$, and

$$
\Lambda=\left(\frac{1}{(\alpha-\mu) \Gamma(\alpha)} \int_{0}^{1}(1-s)^{\alpha-1}(\alpha-\mu+\mu s) q(s) d s\right)^{-1}=\frac{575 \sqrt{\pi}}{32} .
$$

Let $a=1$, then for any $(t, x) \in[0,1] \times[0, a]$, direct computations give

$$
f(t, x) \leq f(t, 1) \leq f(1,1)=11+e^{3}<\frac{575 \sqrt{\pi}}{32}=\Lambda a .
$$

Therefore, all assumptions of Theorem 3.1 are satisfied. Thus Theorem 3.1 ensures that the problem (4.2) has two positive solutions $v^{*}$ and $w^{*}$, satisfying $0<\left\|v^{*}\right\| \leq\left\|w^{*}\right\| \leq 1$ and $\lim _{k \rightarrow \infty}\left\|v_{k}-v^{*}\right\|=0, \lim _{k \rightarrow \infty}\left\|w_{k}-w^{*}\right\|=0$, where

$$
\begin{aligned}
v_{0}(t)=0, \quad t \in[0,1] & \\
v_{k+1}(t)= & \frac{16 t^{7 / 2}}{525 \sqrt{\pi}} \int_{0}^{1}(1-s)^{2}(5+4 s)\left[e^{v_{k}(s)+s+1}+2 v_{k}^{2}(s)+4 s+5\right] d s \\
& -\frac{16}{105 \sqrt{\pi}} \int_{0}^{t} \frac{(t-s)^{7 / 2}}{(1-s)^{3 / 2}}\left[e^{v_{k}(s)+s+1}+2 v_{k}^{2}(s)+4 s+5\right] d s, \\
& t \in[0,1], k=0,1,2, \ldots
\end{aligned}
$$

and

$$
\begin{aligned}
w_{0}(t)=t^{7 / 2}, \quad t \in[0,1], & \\
w_{k+1}(t)= & \frac{16 t^{7 / 2}}{525 \sqrt{\pi}} \int_{0}^{1}(1-s)^{2}(5+4 s)\left[e^{w_{k}(s)+s+1}+2 w_{k}^{2}(s)+4 s+5\right] d s \\
& -\frac{16}{105 \sqrt{\pi}} \int_{0}^{t} \frac{(t-s)^{7 / 2}}{(1-s)^{3 / 2}}\left[e^{w_{k}(s)+s+1}+2 w_{k}^{2}(s)+4 s+5\right] d s, \\
& t \in[0,1], k=0,1,2, \ldots
\end{aligned}
$$

\section{Competing interests}

The authors declare that they have no competing interests.

\section{Authors' contributions}

All authors contributed equally in this article. They read and approved the final manuscript.

\section{Author details}

${ }^{1}$ College of Electron and Information, Zhejiang University of Media and Communications, Hangzhou, Zhejiang 310018 , China. ${ }^{2}$ Department of Mathematics, Shanghai Normal University, Shanghai, 200234, China.

\section{Acknowledgements}

The authors sincerely thanks the anonymous referees for their valuable suggestions and comments which have greatly helped improve this article. The first author was supported financially by the Natural Science Foundation of Zhejiang Province of China (Y12A01012). The second author was supported financially second by the Foundation of Shanghai Natural Science (13ZR1430100) and the Foundation of Shanghai Municipal Education Commission (DYL201105). 


\section{References}

1. Agarwal, RP, Liu, YS, O'Regan, O, Tian, CC: Positive solutions of two-point boundary value problems for fractional singular differential equations. Differ. Equ. 48, 619-629 (2012)

2. Ahmad, B, Ntouyas, SK: A study of higher-order nonlinear ordinary differential equations with four-point nonlocal integral boundary conditions. J. Appl. Math. Comput. 39, 97-108 (2012)

3. Bai, ZB, Sun, WC: Existence and multiplicity of positive solutions for singular fractional boundary value problems. Comput. Math. Appl. 63, 1369-1381 (2012)

4. Bai, ZB, Qiu, TT: Existence of positive solution for singular fractional differential equation. Appl. Math. Comput. 215, 2761-2767 (2009)

5. Cabada, A, Wang, GW: Positive solutions of nonlinear fractional differential equations with integral boundary value conditions. J. Math. Anal. Appl. 389, 403-411 (2012)

6. Goodrich, CS: Existence of a positive solution to a class of fractional differential equations. Appl. Math. Lett. 23, 1050-1055 (2010)

7. Goodrich, CS: Existence of a positive solution to systems of differential equations of fractional order. Comput. Math. Appl. 62, 1251-1268 (2011)

8. Goodrich, CS: Existence and uniqueness of solutions to a fractional difference equation with nonlocal conditions. Comput. Math. Appl. 61, 191-202 (2011)

9. Jiang, WH, Huang, XQ, Guo, WW, Zhang, Q: The existence of positive solutions for the singular fractional differential equation. J. Appl. Math. Comput. 41(1-2), 171-182 (2013)

10. Karakostas, GL: Non-existence of solutions for two-point fractional and third-order boundary-value problems Electron. J. Differ. Equ. 2013, Article ID 152 (2013)

11. Wang, YQ, Liu, LS, Wu, YH: Positive solutions for a nonlocal fractional differential equation. Nonlinear Anal. 74, 3599-3605 (2011)

12. Vong, S: Positive solutions of singular fractional differential equations with integral boundary conditions. Math Comput. Model. 57, 1053-1059 (2013)

13. Wu, JW, Zhang, XG, Liu, LS, Wu, YH: Positive solutions of higher-order nonlinear fractional differential equations with changing-sign measure. Adv. Differ. Equ. 2012, Article ID 71 (2012)

14. Yang, L, Chen, HB: Unique positive solutions for fractional differential equation boundary value problems. Appl. Math Lett. 23, 1095-1098 (2010)

15. Liu, Y, Zhang, WG, Liu, XP: A sufficient condition for the existence of a positive solution for a nonlinear fractional differential equation with the Riemann-Liouville derivative. Appl. Math. Lett. 25, 1986-1992 (2012)

16. Yuan, CJ: Multiple positive solutions for $(n-1,1)$-type semipositone conjugate boundary value problems of nonlinear fractional differential equations. Electron. J. Qual. Theory Differ. Equ. 2010, Article ID 36 (2010)

17. Yuan, CJ: Two positive solutions for $(n-1,1)$-type semipositone integral boundary value problems for coupled systems of nonlinear fractional differential equations. Commun. Nonlinear Sci. Numer. Simul. 17, 930-942 (2012)

18. Zhai, CB, Hao, MR: Mixed monotone operator methods for the existence and uniqueness of positive solutions to Riemann-Liouville fractional differential equation boundary value problems. Bound. Value Probl. 2013, Article ID 85 (2013)

19. Zhang, SQ: The existence of a positive solution for a nonlinear fractional differential equation. Comput. Math. Appl. 59, 1300-1309 (2010)

20. Zhang, XG, Han, YF: Existence and uniqueness of positive solutions for higher order nonlocal fractional differential equations. Appl. Math. Lett. 25, 555-560 (2012)

21. Zhang, XG, Liu, LS, Wu, YH: Multiple positive solutions of a singular fractional differential equation with negatively perturbed term. Math. Comput. Model. 55, 1263-1274 (2012)

22. Zhang, XG, Liu, LS, Wu, YH, Lu, YN: The iterative solutions of nonlinear fractional differential equations. Appl. Math. Comput. 219, 4680-4691 (2013)

23. Ma, DX, Yang, XZ: Existence and iteration of positive solutions to third order three-point BVP with increasing homeomorphism and positive homomorphism. Rocky Mt. J. Math. 43, 539-550 (2013)

24. Sun, B, Ge, WG: Successive iteration and positive pseudo-symmetric solutions for a three-point second-orde p-Laplacian boundary value problems. Appl. Math. Comput. 188, 1772-1779 (2007)

25. Sun, YP, Zhang, XP, Zhao, M: Successive iteration and positive solutions for fourth-order two-point boundary value problems. Abstr. Appl. Anal. 2013, Article ID 621315 (2013)

26. Yao, QL: Successively iterative technique of a classical elastic beam equation with Carathéodory nonlinearity. Acta Appl. Math. 108, 385-394 (2009)

27. Zhang, XP: Existence and iteration of monotone positive solutions for an elastic beam equation with corner. Nonlinear Anal., Real World Appl. 10, 2097-2103 (2009)

28. Kilbas, AA, Srivastava, HM, Trujillo, Jj: Theory and Applications of Fractional Differential Equations. North-Holland Mathematics Studies, vol. 204. Elsevier, Amsterdam (2006)

29. Podlubny, I: Fractional Differential Equations. Math. Sci. Eng., vol. 198. Academic Press, San Diego (1999)

10.1186/1687-1847-2014-29

Cite this article as: Sun and Sun: Positive solutions and monotone iterative sequences for a fractional differential equation with integral boundary conditions. Advances in Difference Equations 2014, 2014:29 